\title{
The Comprehensive Evaluation and Study Based on National Happiness Index System
}

\author{
Dongxiao Niu, Qiaoling Wu, Leilei Fan, Chunxiang Liu \\ School of Economics and Management, North China Electric Power University, Beijing, China \\ wuqiaoling7@163.com
}

\begin{abstract}
Happiness index is used to measure people's satisfaction with the social and economic development. In this paper seven typical cities in Yangtze River delta are selected as the research object, and eleven variables from five aspects are selected to establish the comprehensive index system of residents' happiness index. Then the paper determines the optimal weight of index by combining subjective and objective method. It evaluates the residents' happiness index about this seven cities by using TOPSIS, the Rumania selection method and the project queuing method based on evaluating relative position. At last the paper analyzes the evaluation results, and draws a conclusion that corresponds with the actual situation.

Index Terms - comprehensive evaluation, happiness index, index system
\end{abstract}

\section{Introduction}

What is happiness? One of the founders of economics Jeremy Bentham said, people feel delighted and comfortable when their desires are met fully or partly, the feeling is happiness[1]. Paul A.Samuelson, master of modern economics, constructed a happiness equation, happiness = utility/desire[2]. Now more and more scholars at home and abroad are researching the issue of happiness. Doctor Xing evaluates happiness from the perspective of psychology and uses a large amount of subjective well-being measure scale in the study[3]. Zhou Sijun says that national happiness index is influenced by economy, environment, population and society[4]. In foreign countries, Bhutan is the first country to introduce happiness index instead of GDP. It pays more attention to the mental health status of the residents, not just the GDP growth[5].

\section{The establishment of the evaluation index system}

According to the existing research of national happiness index, this paper established the national happiness index system considering six important principles: purposiveness, scientificalness, systematicness, operability, comparability and combination of qualitative and quantitative analysis. The index system(Table1) includes five aspects : economic income, employment and social security, education and medical service, culture and sports, living environment[6].

\section{The evaluation method}

\section{A. Topsis}

The standard TOPSIS method attempts to choose alternatives that simultaneously have the shortest distance from the positive ideal solution and the farthest distance from the negative-ideal solution. The positive ideal solution maximizes the benefit criteria and minimizes the cost criteria, whereas the negative ideal solution maximizes the cost criteria and minimizes the benefit criteria[7].

Table 1 The evaluation index system based on national happiness index

\begin{tabular}{|c|c|c|}
\hline \multirow{11}{*}{$\begin{array}{c}\text { The comprehensive evaluation } \\
\text { based on national happiness } \\
\text { index system }\end{array}$} & \multirow{3}{*}{ economic income $\left(x_{1}\right)$} & Disposable income of urban residents $\left(x_{11}\right)$ \\
\hline & & Per capita net income of rural residents $\left(x_{12}\right)$ \\
\hline & & Engel's coefficient of urban residents consumption $\left(x_{13}\right)$ \\
\hline & \multirow{2}{*}{$\begin{array}{l}\text { employment and social } \\
\operatorname{security}\left(x_{2}\right)\end{array}$} & Registered urban unemployment rate $\left(x_{21}\right)$ \\
\hline & & Contributors in five major insurance $\left(x_{22}\right)$ \\
\hline & \multirow[b]{2}{*}{ education and medical service $\left(x_{3}\right)$} & Education penetration $\left(x_{31}\right)$ \\
\hline & & $\begin{array}{l}\text { Number of doctors and nurses per one thousand } \\
\qquad \operatorname{people}\left(x_{32}\right)\end{array}$ \\
\hline & \multirow{2}{*}{ culture and sports $\left(x_{4}\right)$} & The number of books in the library $\left(x_{41}\right)$ \\
\hline & & The number of gymnasiums $\left(x_{42}\right)$ \\
\hline & \multirow{2}{*}{ living environment $\left(x_{5}\right)$} & Per capita public green area $\left(x_{51}\right)$ \\
\hline & & Production accident deaths per 100000 people $\left(x_{52}\right)$ \\
\hline
\end{tabular}


1) There are $n$ evaluation objects and $m$ evaluation indexes. Construct normalized decision matrix $Z_{i j}=x_{i j} / \sqrt{\sum_{i=1}^{n} x_{i j}^{2}}(i=1,2, \ldots ., m \quad \mathrm{j}=1,2, \ldots ., n)$ where $x_{i j}$ and $Z_{i j}$ are original and normalized matrix respectively.

2) Determine the positive ideal and negative ideal solutions. The positive ideal solution is $Z^{+}=\max \left(Z_{1 j}^{+}, Z_{2 j}^{+}, \ldots Z_{m j}^{+}\right)$, while the negative ideal solution is $Z^{-}=\min \left(Z_{1 j}^{-}, Z_{2 j}^{-}, \ldots Z_{m j}^{-}\right)$.

Calculate the separation measures for each alternative. The separation from positive ideal alternative is $\mathrm{D}_{\mathrm{i}}^{+}=\sqrt{\sum_{j=1}^{\mathrm{m}}\left(\mathrm{Z}_{\max }-\mathrm{Z}_{\mathrm{ij}}\right)^{2}}$, and the separation from negative ideal alternative is $\mathrm{D}_{\mathrm{i}}^{-}=\sqrt{\sum_{j=1}^{\mathrm{m}}\left(\mathrm{Z}_{\text {minj }}-\mathrm{Z}_{\mathrm{ij}}\right)^{2}}$.

Calculate the relative closeness to the ideal solution $C_{i}=D_{i}^{-} / D_{i}^{+}+D_{i}^{-}$. Select the alternative with $C_{i}$ closest to 1 .

\section{B. The Rumania selection method}

The Rumania selection method converts the score of each index into a scale of 0 to 100 score, which is called standardization. Compare the score of each solution from the various indexes respectively. The best solution score is 100 , the worst solution score is 1 , and the middle solution score is $X=\frac{99 *(C-B)}{A-B}+1$, where $\mathrm{A}$ is the value of the best solution index, $\mathrm{B}$ is the value of the worst solution index, $\mathrm{C}$ is the value of the middle solution index, $\mathrm{X}$ is the score of the middle solution.

\section{The project queuing method based on evaluating relative position}

First the project queuing method based on evaluating relative position is to make the precedence relation of each solution expressed with 0-1 matrix. Then queue up the solutions synthetically. Here is the procedure in detail.

1) The decision maker gives the weight of each attribute of $j$.

2) Compare the attribute of $j$ with each other, and give the precedence relation matrix.

a. If the value of attribute $\mathrm{j}$ of $X_{i}$ is better than the value of attribute $\mathrm{j}$ of $X_{k}$, then mark it as $X_{i} \succ X_{k}$.

b. If the value of attribute $\mathrm{j}$ of $X_{i}$ is worse than the value of attribute $\mathrm{j}$ of $X_{k}$, then mark it as $X_{i} \prec X_{k}$.

c. If the value of attribute $\mathrm{j}$ of $X_{i}$ is similar with the value of attribute $\mathrm{j}$ of $X_{k}$, then mark it as $X_{i} \sim X_{k}$.
3) Determine the relationship of the pair of solutions $\left(X_{i}, X_{k}\right)$.

a. Calculate the weight of the pair of solutions $\left(X_{i}, X_{k}\right)$.Add the value of attribute $\mathrm{j}$ of $X_{i}$ up to the value of attribute $\mathrm{j}$ of $X_{k}$ respectively, and mark it as $w\left(X_{i} \succ X_{k}\right) \cdot w\left(X_{i} \prec X_{k}\right)$ and $\mathrm{w}\left(\mathrm{X}_{\mathrm{i}} \sim \mathrm{X}_{\mathrm{k}}\right)$ are similar to it.

$$
\begin{aligned}
& w\left(X_{i} \succ X_{k}\right)=\sum_{j \in\left(X_{i} \succ X_{k}\right)_{j}} w_{j} \\
& w\left(X_{i} \prec X_{k}\right)=\sum_{j \in\left(X_{i} \prec X_{k}\right)_{j}} w_{j} \\
& w\left(X_{i} \sim X_{k}\right)=\sum_{j \in\left(X_{i} \sim X_{k}\right)_{j}} w_{j}
\end{aligned}
$$

b. Calculate the indicated value of the pair of solutions ( $\left.X_{i}, X_{k}\right)$, and mark it as $A_{\sigma}\left(X_{i}, X_{k}\right)$.

$$
A_{\sigma}\left(X_{i}, X_{k}\right)=\frac{w\left(X_{i} \succ X_{k}\right)+\sigma w\left(X_{i} \sim X_{k}\right)}{w\left(X_{i} \prec X_{k}\right)+\sigma w\left(X_{i} \sim X_{k}\right)}
$$

In the formula, the value of $\sigma$ reflect the importance of $X_{i}$ and $X_{k}$ in the decision-making process where $0 \leq \sigma \leq 1$.

c. Select the threshold $A \geq 1$, and determine if the solution is good or bad.

$$
\left\{\begin{array}{c}
X_{i} \succ X_{k}, \text { if } A_{\sigma}\left(X_{i}, X_{k}\right) \geq A \\
X_{i} \sim X_{k}, \text { if } \frac{1}{A}<A_{\sigma}\left(X_{i}, X_{k}\right)<A \\
X_{i} \prec X_{k}, \text { if } A_{\sigma}\left(X_{i}, X_{k}\right) \leq \frac{1}{A}
\end{array}\right.
$$

d. According to the evaluation of the solutions, we can draw the overall figure about strengths and weaknesses or relational tables about priority of each solution. Calculate the indicated value of each solution, and rank the solutions with the indicated value.

\section{A case study}

In this paper, we select seven typical cities in Yangtze River delta as the research object and evaluate the residents' happiness index about these seven cities by different methods. Then we analyze the results and compare the result of different method.

The result of the TOPSIS method is as Table 2. 
Table 2 The result of the TOPSIS method

\begin{tabular}{|l|c|c|c|c|c|c|c|}
\hline & WuXi & YangZhou & NanJing & HeFei & TaiZhou & NingBo & WenZhou \\
\hline $\begin{array}{l}\text { The separation from } \\
\text { positive ideal alternative }\end{array}$ & 0.0048 & 0.0088 & 0.0010 & 0.0142 & 0.02289 & 0.0108 & 0.0172 \\
\hline $\begin{array}{l}\text { The separation from } \\
\text { negative ideal alternative }\end{array}$ & 0.0120 & 0.0114 & 0.0246 & 0.0068 & 0.0014 & 0.0107 & 0.0032 \\
\hline the indicated value & 0.7143 & 0.5644 & 0.9609 & 0.3246 & 0.0576 & 0.4977 & 0.1569 \\
\hline rank & 2 & 3 & 1 & 5 & 7 & 4 & 6 \\
\hline
\end{tabular}

Table 3 The weighted comprehensive scores of the Rumania selection method

\begin{tabular}{|c|c|c|c|c|c|c|c|}
\hline & WuXi & YangZhou & NanJing & HeFei & TaiZhou & NingBo & WenZhou \\
\hline $\begin{array}{c}\text { The weighted } \\
\text { comprehensive scores }\end{array}$ & 96.32 & 72.24 & 116.25 & 41.30 & 45.91 & 86.80 & 61.53 \\
\hline
\end{tabular}

Table 4 The precedence relation matrix

\begin{tabular}{|c|c|c|c|c|c|c|c|}
\hline & $\begin{array}{c}\text { WuXi } \\
\left(X_{1}\right)\end{array}$ & $\begin{array}{c}\text { YangZhou } \\
\left(X_{2}\right)\end{array}$ & $\begin{array}{c}\text { NanJing } \\
\left(X_{3}\right)\end{array}$ & $\begin{array}{c}\text { HeFei } \\
\left(X_{4}\right)\end{array}$ & $\begin{array}{c}\text { TaiZhou } \\
\left(X_{5}\right)\end{array}$ & $\begin{array}{c}\text { NingBo } \\
\left(X_{6}\right)\end{array}$ & $\begin{array}{c}\text { WenZhou } \\
\left(X_{7}\right)\end{array}$ \\
\hline WuXi $\left(X_{1}\right)$ & 1 & 1 & 0 & 1 & 1 & 1 & 1 \\
\hline $\begin{array}{c}\text { YangZhou } \\
\left(X_{2}\right)\end{array}$ & 0 & 1 & 0 & 1 & 1 & 1 & 1 \\
\hline NanJing $\left(X_{3}\right)$ & 1 & 1 & 1 & 1 & 1 & 0 & 0 \\
\hline HeFei $\left(X_{4}\right)$ & 0 & 0 & 0 & 1 & 1 & 1 & 0 \\
\hline TaiZhou $\left(X_{5}\right)$ & 0 & 0 & 0 & 0 & 1 & 1 & 1 \\
\hline NingBo $\left(X_{6}\right)$ & 1 & 1 & 0 & 1 & 1 & 0 & 1 \\
\hline WenZhou & 0 & 0 & 0 & 1 & & 0 \\
\hline
\end{tabular}

According to the result above, the rank of the seven cities about the national happiness index is

\section{NanJing $>$ WuXi $>$ YangZhou $>$ NingBo $>$ HeFei $>$ WenZhou $>$ TaiZhou .}

The result of the Rumania selection method is as Table 3.

According to the result above, the rank of the seven cities about the national happiness index is

NanJing $>$ WuXi $>$ NingBo $>$ YangZhou $>$ WenZhou $>$ TaiZhou $>$ HeFei .

The result of the project queuing method based on evaluating relative position is as Table 4 .

According to the precedence relation matrix, calculate the indicated value of each solution. The result is as follows:

$V_{1}=3, V_{2}=1, V_{3}=6, V_{4}=-4, V_{5}=-6, V_{6}=2, V_{7}=-2$. The rank of the seven cities about the national happiness index is

NanJing $>$ WuXi $>$ NingBo $>$ YangZhou $>$ WenZhou $>$ HeFei $>$ TaiZhou .

\section{Conclusions}

The evaluation methods evaluate the differences of each evaluation object comprehensively. So the difference is greater, the result is better. The difference by the Rumania selection method and the project queuing method based on evaluating relative position is greater than that by the TOPSIS method. The rank of WuXi and NanJing is the same by these three methods. NanJing is NO.1, WuXi is NO.2, and the others are not in accordance with each other by different methods.

\section{References}

[1] Jeremy Bentham, "An introduction to principle of morals and legislation," Shaanxi People's Publishing House, 2009.

[2] ZengHong, Zhao Minglong. "The establishment and comprehensive evaluation of Urban residents' happiness index system," Commercial Times, Vol.14, p.19-21, 2012.

[3] Xing Zhanjun. "The research overview of subjective well-being measurement," Psychological Science, Vol.25, p.336-338, 2002.

[4] Zhou Sijun. "The measure and evaluation of national happiness index in Hunan province," Consumer Economics, Vol.24, p.90-93, 2008.

[5] Luo Min, "The research of index system of urban residents' happiness index measure," Chongqing Technology and Business University, 2012.

[6] Niu Song. "The research to indicators system of national happiness index." Nanjing University of Finance and Economics, 2009.

[7] Chi Guotai, Zhao Guangjun, Li Gang. "The Evaluation Model of Society Development Based on Approximating Ideal Point and G1 Method," Systems Engineering, Vol. 27, p.44-50, 2009. 